Ann. Génśt. Sél. anim., I969, 1 (4), 39I-395.

\title{
SURPROLIFICITÉ DES GÉNOTYPES SANS CORNES DANS LES RACES CAPRINES ALPINE SAANEN, ALPINE CHAMOISÉE ET POITEVINE
}

\author{
G. RICORDEAU \\ avec la collaboration technique de J. Boulllon (*), M. Carpentier, \\ P. Guillimin et A. Lajous \\ Station de Génétique quantitative et appliquée, \\ Centre national de Recherches zootechniques, 78-Jouy-en-Josas \\ Institut national de la Recherche agronomique
}

\section{RÉSUMÉ}

Les boucs homozygotes sans cornes $(P P)$ sont significativement plus prolifiques que les boucs hétérozygotes sans cornes $(P p)$ et que les boucs cornus $(p p)$.

Il serait intéressant de savoir comment cette surprolificité se rattache aux atteintes épididymaires qui affectent une partie des mâles de même génotype, les rendant parfois stériles.

\section{INTRODUCTION}

Dès I964, SOLLER et KEMPENICK ont montré que les chèvres mottes avaient un taux de prolificité supérieur à celui des chèvres cornues. Nous avons confirmé ultérieurement ce fait et mis en évidence une influence probable du mâle (RIcoRDEAU et LAUVERGNE, I967). C'est cette influence que nous nous proposons de vérifier grâce à l'ensemble des observations recueillies de I965 à r969.

\section{I. - MATÉRIEL ET MÉTHODE}

Les données proviennent tout d'abord de la zone du syndicat caprin de Sainte-Croix-Vallée Française (Lozère) sur. 2919 chèvres de race Saanen contrôlées en fermes ou en station de testage, au cours de 5 campagnes successives (1965 à 1969). Des observations, moins nombreuses, ont été

(*) Directeur de la Station de testage caprin, 48-Moissac. 
recueillies de 1967 à 1969 au Domaine expérimental de Galle (Cher) sur 62 chèvres Saanen, 255 chamoisées et 120 poitevines. Les données utilisables comprennent au total 3356 mises-bas et 5952 produits.

La détermination du cornage des boucs mottes (PP ou $\mathrm{Pp}$ ) a été faite d'après les résultats de testage. Pour les types d'accouplements, nous utiliserons la classification adoptée par LAOR et al. (1962).

Au cours de ces 5 campagnes, 126 boucs ont été utilisés dont 107 de race Saanen (13 de génotype $P P, 72 P p$ et $22 p p), 12$ de race chamoisée $(2 P p$ et $10 p p)$ et 7 poitevins $(5 P p$ et $2 p p)$.

Le taux de prolificité est exprimé par le nombre total de produits obtenus pour 100 mères qui ont mis bas.

Au cours des 2 dernières campagnes (1968 et 69 ), on a pratiqué sur une partie du cheptel les saillies ou inséminations après synchronisation des chaleurs à l'aide d'éponges vaginales imprégnées d'acétate de fluorogestone, mais cette technique a été utilisée sans tenir compte du cornage des boucs, aussi nous n'avons pas fait de distinction entre les résultats obtenus.

Pour tenir compte des différences dues à l'année, à l'âge des chèvres, au troupeau ou à la race, nous avons calculé, pour chaque catégorie d'animaux, les taux de prolificité des 6 types d'accouplements, puis nous les avons comparés deux à deux et testé la signification des différences en utilisant le test $t$ défini précédemment (RICORDEAU, 1967); pour chaque comparaison, la différence $d_{i}$ est pondérée par le coefficient $w_{i}$ fonction du nombre de mises-bas $n_{1}$ et $n_{2}$ dans chaque groupe $\left(w_{l}=n_{1} n_{2} /\left(n_{1}+n_{2}\right)\right), k$ étant le nombre de comparaisons effectuées.

$$
t=\frac{\bar{d} \sqrt{\sum w_{i}}}{\sqrt{\frac{\sum w_{i} d_{i}^{2}-\frac{\left(\sum w_{i} d_{i}\right)^{2}}{\sum w_{i}}}{k-1}}}
$$

Les boucs homozygotes sans cornes $P P$ étant uniquement de race Saanen, les comparaisons effectuées avec les types d'accouplements 1 et 4 concernent donc uniquement cette seule race.

\section{II. - RÉSULTATS}

Le tableau I donne le résultat brut de prolificité des 6 types d'accouplements, mais ces valeurs n'ont évidemment qu'une valeur indicative.

TABLEAU I

Niveau moyen de prolificité dans les 6 types d'accouplements (moyennes brutes de l'ensemble des données de 3 races sur 5 années)

\begin{tabular}{|c|c|c|c|c|c|c|}
\hline \multicolumn{2}{|r|}{$\begin{array}{c}\text { Types } \\
\text { d'accouplements }\end{array}$} & \multicolumn{2}{|c|}{ Nombre de } & \multirow{2}{*}{$\begin{array}{l}\text { Taux } \\
\text { de } \\
\text { prolificité }\end{array}$} & \multicolumn{2}{|c|}{$\begin{array}{c}\text { Nombre } \\
\text { de femelles vraies } \\
\text { pour roo mises-bas }\left({ }^{1}\right)\end{array}$} \\
\hline No & Père Mère & Mises-bas & $\begin{array}{c}\text { Chevreaux } \\
\text { nés }\end{array}$ & & Théorique & Observé \\
\hline $\begin{array}{l}\text { I } \\
2 \\
3 \\
4 \\
5 \\
6\end{array}$ & $\begin{array}{c}P P \times P p \\
P p \times P p \\
p p \times P p \\
P P \times p p \\
P p \times p p \\
p p \times p p \\
\text { Total général . . }\end{array}$ & $\begin{array}{r}263 \\
\text { I I } 86 \\
790 \\
\text { I } 49 \\
548 \\
420 \\
\\
3356\end{array}$ & $\begin{array}{r}500 \\
2074 \\
1472 \\
264 \\
944 \\
698 \\
595^{2}\end{array}$ & $\begin{array}{l}190,11 \\
174,88 \\
186,32 \\
177,19 \\
172,26 \\
166,19 \\
177,35\end{array}$ & $\begin{array}{l}47,5 \\
65,6 \\
93,2 \\
88,6 \\
86, \mathrm{I} \\
83, \mathrm{r} \\
\end{array}$ & $\begin{array}{l}46,8 \\
68,2 \\
86,9 \\
93,1 \\
82,9 \\
80,4 \\
-\end{array}$ \\
\hline
\end{tabular}

(1) Les proportions théoriques de femelles vraies sont de 25 dans le type $1,37,5$ dans le type 2 et 50 dans les types 3 à 6 . Les proportions réellement observées sont respectivement de $24,6-39,0-46,6-52,5-48,1$ et 48,4 pour les accouplements $I$ à 6 . 
TABLEAU 2

Comparaisons deux à deux du taux de prolificité des reproducteurs dans différents accouplements

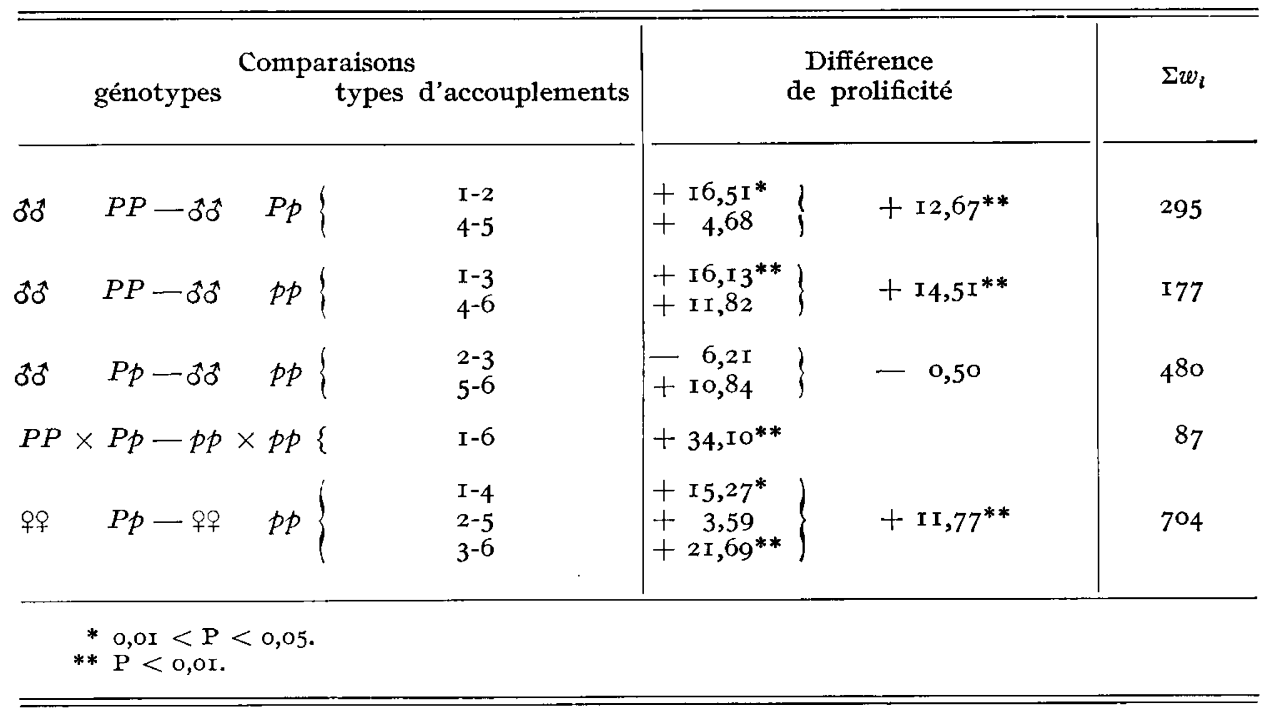

Dans le tableau 2, nous avons comparé 2 à 2 les différents types d'accouplements (suivant la méthode décrite précédemment) et calculé la signification des différences.

\section{III. - DISCUSSION}

\section{Surprolificité des femelles}

Nous retrouvons tout d'abord la différence de prolificité entre les chèvres mottes hétérozygotes $\mathrm{P} p$ et les chèvres cornues $p p$ déjà signalée par SoLLER et KEMPENICH (I964). Le coefficient de surprolificité des femelles $\mathrm{P} p$ par rapport aux femelles $p p$ s'établissait à $\mathrm{I}, 062$ pour les Israéliens; nous le trouvons ici égal à I,069.

\section{Surprolificité des mâles}

En comparant les accouplements I et 2, 4 et 5 d'une part, I et 3, 4 et 6 d'autre part, dans lesquels les femelles sont de même génotype, on constate une surprolificité significative des boucs mottes $\mathrm{PP}$ par rapport aux boucs mottes $\mathrm{P} p(+\mathrm{I} 2,7$ produits $p$. Ioo mises-bas) et aux boucs cornus $p p(+\mathrm{I} 4,5)$; en revanche, il n'existe aucune différence significative de prolificité entre les boucs $\mathrm{P} p$ et $p p$. La différence la plus grande $(+34, \mathrm{I})$ est celle qui existe entre les types d'accouplements I et 6 , l'accouplement entre animaux cornus étant nettement le moins prolifique.

Compte tenu du taux de prolificité moyen des accouplements 2 et 5 d'une part (I74 produits pour roo mises-bas, tabl. I), des accouplements 3 et 6 d'autre part (I79), le coefficient de surprolificité des boucs homozygotes sans cornes PP est de $\mathrm{I}, 073$ par rapport aux boucs $\mathrm{P} p$ et de $\mathrm{I}, 080$ par rapport aux boucs $p p$ : ces deux coefficients ne sont pas significativement différents. 


\section{Essai d'interprétation}

Pressentie en I967 (RICORDEAU et LAUVERGNE, I967), cette surprolificité des mâles semble maintenant définitivement prouvée. On peut se demander à quoi elle est due et d'où elle provient.

Un examen systématique du sperme des boucs de différents génotypes, dans les conditions de l'insémination artificielle, permettrait peut-être de mettre en évidence des différences dans la qualité de la semence. Chez les volailles, par exemple, Cochez (I95I) puis Petritjean et Cochez (I966) ont montré que les coqs homozygotes à crête rosacée $\mathrm{RR}$ ont un taux de fertilité inférieur d'environ 20 p. Ioo à celui des coqs $\mathrm{R} r$ ou $r r$ à crête simple et possèdent un sperme dont la motilité des spermatozoïdes est moins bonne après conservation.

Pour expliquer l'origine de cette surprolificité, on peut essayer de faire le rapprochement de l'action du gène $P$ dans les deux sexes.

A l'état hétérozygote, l'action du gène $P$ est limitée : elle est probablement nulle chez les mâles et entraîne une surprolificité de 6 à 7 p. roo des femelles $P p$ par rapport aux femelles $p p$.

A l'état homozygote, en revanche, toutes les femelles PP sont masculinisées avec expressivité variable (de la femelle stérile au pseudo mâle) et les mâles présentent différentes particularités. Certains sont atteints d'obstruction partielle ou totale de 1'épididyme, ce qui entraîne dans ce dernier cas la stérilité (cf. LAUVERGNE, I969 pour revue de 1'action du gène); les autres manifestent une surprolificité de 7 à $8 \mathrm{p}$. Ioo par rapport aux boucs $\mathrm{P} p$ ou $p p$.

Il serait évidemment très intéressant de savoir si cet accroissement de prolificité des boucs PP est le résultat du même phénomène qui entraîne un développement anormal des épididymes : peut-être un excès d'androgènes, ou plus vraisemblablement une sensibilité plus grande aux stéroïdes mâles des animaux de génotype PP. Cela mérite un examen approfondi des qualités de reproduction des mâles de génotype sans cornes.

\section{Aspect pratique}

Le $6^{\mathrm{e}}$ type d'accouplement du tableau I ( $\sigma^{\star} \sigma^{\star}$ cornus $\times$ 우 cornues) est le moins intéressant en ce qui concerne le nombre de produits par mise-bas. Mais du point de vue production de femelles vraies, ce type d'accouplement est quand même plus intéressant que le type I, le plus prolifique. En effet, le type I donne en moyenne I9o chevreaux pour Ioo mises-bas, mais seulement $25 \mathrm{p}$. Ioo de femelles vraies, soit 47 femelles; avec le type 6 , nous obtenons I66 produits et 83 femelles en moyenne (tabl. I). Du point de vue production de femelles, si l'on exclut 1'utilisation de mâles PP, les types d'accouplement les plus favorables sont les accouplements réciproques $n^{\circ \mathrm{s}} 3\left(\sigma^{*} \sigma^{*} p p \times\right.$ 우 $\mathrm{P} p$ ) et $5\left({ }^{*} \sigma^{*} \mathrm{P} p \times\right.$ 우 $p p$ ) avec lesquels on peut espérer théoriquement 93 et 86 produits femelles pour roo mises-bas. Pratiquement, compte tenu des variations dans le rapport des sexes, le type 6 est presque aussi avantageux ( 80 femelles avec le type 6 contre 87 et 83 femelles pour les types 3 et 5 ). Ces trois accouplements peuvent être programmés en ferme avec 2 boucs ou, plus aisément, grâce à l'insémination artificielle. 


\title{
REMERCIEMENTS
}

Nous tenons à remercier MM. MÉrat et Lauvergne de leurs conseils dans l'élaboration de cette note.

\section{SUMMARY}

\author{
SURPROLIFICITY OF POLLED GENOTYPES IN ALPINE SAANEN \\ $A L P I N E$ CHAMOISEE AND POITEVINE GOAT BREEDS
}

The prolificity rate (total of the products of 100 kiddings) of 6 possible types of matings between horned or polled rams $(P P, P p$ and $p p)$ has been compared with polled or horned females $(P p$ and $p p)$. The comparison is based on observations gathered in farms and station during 5 reproduction periods (3 356 kiddings, 5952 products) using 126 rams of the Saanen, chamoisée and poitevine breeds.

It was verified that polled goats $(\mathrm{Pp})$ are, on an average, more prolific than horned $p p$ goats (surprolificity coefficient of 1.069). We also were able to confirm a phenomenon already observed by us; namely, that homozygous polled $P P$ rams are more prolific than heterozygous polled $P p$ rams or horned $p p$ rams (surprolificity coefficients of 1.073 and 1.080 , respectively; Table 2 ). There is no significant difference in the prolificity between $P p$ and $p p$ rams.

Better prolificity in $P P$ males is perhaps due to higher sperm quality, such as may be measured, but these examinations have not yet been done. It would be interesting to know if there is a relationship between the sperm and epididymal impairment, which seems the result of homozygous $P$ gene action on genetical males.

The most prolific type of mating is mating 1 ( $\sigma^{\star} P P \times$ 우 $P p$ ), but matings which should produce a maximum of true females for reproduction are the reciprocal matings $3\left(\delta^{\star} \delta p p \times\right.$ o $p P p$ ) and $5($ đึ่ $P p \times$ 웅 $P p)$.

\section{RÉFÉRENCES BIBLIOGRAPHIQUES}

Cochez L. P., r95I. Un facteur d'infertilité, équilibré par la sélection, chez la Wyandotte blanche. IX World's Poultry Congr., éd. franç., 1, 8I-88.

I AOR M., B.ARnea A., ANGel H., Soller M., I962. Polledness and hermaphroditism in Saanen goats. Israel J. agric. Res., 12, 83-88.

Lauvergne J. J., 1969. Progrès des connaissances génétiques sur l'intersexualité associée à l'absence de cornes chez la chèvre d'origine alpine. Ann. Génét. Sél. anim., 1, 403-4I2.

Petitjean M. J., Cochez L. P., I966. A propos de la subfertilité des coqs homozygotes pour le gène : $R$ " (crête rosacée). XIII World's Poultry Congr., éd. franç., 125-130.

Ricordeau G., I967. Hérédité des pendeloques en race Saanen. Différence de fécondité entre les génotypes avec ou sans pendeloques. Ann. Zootech., 16, 263-270.

RicordeaU G., IAAUVERGNe J. J., I967. Hypothèse génétique unique pour expliquer la présence d'intersexués, de mâles en excès et de mâles stériles en race caprine Saanen. Ann. Zootech., 16, 323-334.

Soller M., Kempenich O., I964. Polledness and litter size in Saanen Goats. J. Hered., 55, 301-304. 